\title{
Dynamic admittance of carbon nanotube-based molecular electronic devices and their equivalent electric circuit
}

\author{
ChiYung Yam ${ }^{1}$, Yan Mo ${ }^{1}$, Fan Wang ${ }^{1}$, Xiaobo $\mathrm{Li}^{1}$, \\ GuanHua Chen ${ }^{1}$, Xiao Zheng ${ }^{1,2}$, Yuki Matsuda ${ }^{3}$, \\ Jamil Tahir-Kheli ${ }^{3}$ and William A Goddard III $^{3}$ \\ ${ }^{1}$ Department of Chemistry, Centre of Theoretical and Computational Physics, \\ University of Hong Kong, Hong Kong \\ ${ }^{2}$ Department of Chemistry, Hong Kong University of Science and Technology, Hong Kong \\ ${ }^{3}$ Materials and Process Simulation Center, MC 139-74, California Institute of Technology, \\ Pasadena, CA 91125, USA \\ E-mail: ghc@everest.hku.hk, xzheng@yangtze.hku.hk and wag@wag.caltech.edu
}

Received 18 July 2008, in final form 15 October 2008

Published 18 November 2008

Online at stacks.iop.org/Nano/19/495203

\begin{abstract}
We use first-principles quantum mechanics to simulate the transient electrical response through carbon nanotube-based conductors under time-dependent bias voltages. The dynamic admittance and time-dependent charge distribution are reported and analyzed. We find that the electrical response of these two-terminal molecular devices can be mapped onto an equivalent classical electric circuit and that the switching time of these end-on carbon nanotube devices is only a few femtoseconds. This result is confirmed by studying the electric response of a simple two-site model device and is thus generalized to other two-terminal molecular electronic devices.
\end{abstract}

(Some figures in this article are in colour only in the electronic version)

\section{Introduction}

As the semiconductor industry follows the Moore's law roadmap down to the scale of $20 \mathrm{~nm}$, it becomes important to understand the dynamic response of nanometer-scale molecular electronic devices [1-14]. This requires the use of quantum mechanics to ensure the proper treatment of transient and quantum effects. For practical use by design engineers, it is crucial to cast these quantum effects into the form of classical electric circuits. An important question is whether such a mapping is possible, and if so, what are the forms of the equivalent circuits?

As potentially important components of next-generation integrated circuits, carbon nanotubes (CNTs) have been studied extensively [3-9, 15]. High frequency electrical responses of micrometer-long individual and bundled CNTs have been measured [5], and equivalent electric circuits have been proposed [5, 8, 9]. In this work, we concentrate on a nanometer-scale CNT-based electronic device, and apply first-principles quantum mechanics $(\mathrm{QM})$ to determine its dynamic electrical response. The system of interest is a $(5,5) \mathrm{CNT}(0.68 \mathrm{~nm}$ in diameter and $0.62 \mathrm{~nm}$ in length) which is covalently bonded between two aluminum electrodes, and shown in figure 1 .

\section{Methodology}

To simulate the transient electrical currents through such a molecular device, we employ the rigorous time-dependent density functional theory (TDDFT) that we developed recently [16]. Our theory uses a closed equation of motion (EOM) for the reduced single-electron density matrix of the molecular device $\mathrm{D}, \sigma_{\mathrm{D}}$, as follows:

$$
\mathrm{i} \dot{\sigma}_{\mathrm{D}}=\left[h_{\mathrm{D}}\left[\mathbf{r}, t ; \rho_{\mathrm{D}}(\mathbf{r}, t)\right], \sigma_{\mathrm{D}}\right]-\mathrm{i} \sum_{\alpha=\mathrm{L}, \mathrm{R}} Q_{\alpha}\left[\mathbf{r}, t ; \rho_{\mathrm{D}}(\mathbf{r}, t)\right],
$$




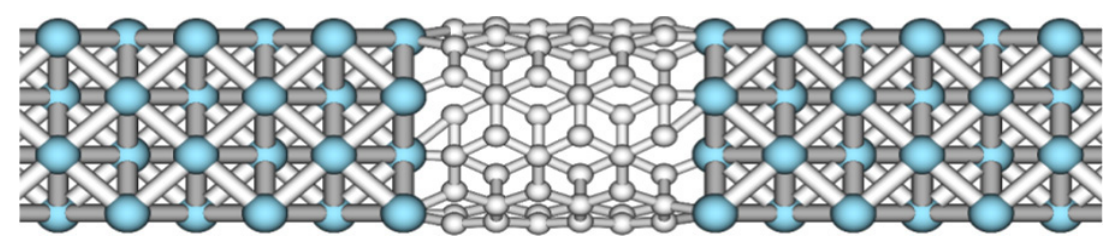

Figure 1. Prototype used for explicit QM calculations of a CNT-based conductor. The (5, 5) CNT device with aluminum as electrodes.

where $h_{\mathrm{D}}(t)$ is the Kohn-Sham Fock matrix for device D. $Q_{\mathrm{L}}\left(Q_{\mathrm{R}}\right)$ is the dissipative term due to the left (right) electrode and is in principle a functional of the time-dependent electron density of device $\mathrm{D}, \rho_{\mathrm{D}}(\mathbf{r}, t)$. The wide band limit approximation $[17,18]$ is adopted in the calculation of $Q$, where the bandwidths of levels in the leads are assumed to be infinitely large and their line widths $\Lambda$ (imaginary part of self-energy $\Sigma^{a}$ ) are treated as energy independent [16].

In the transient regime, it is essential to define the timedependent Fermi energy for an electrode in the presence of an external voltage unambiguously. For a noninteracting electrode, the time-dependent Schrödinger equation for the $i$ th single-electron wavefunction is

$$
\mathrm{i} \frac{\partial\left|\psi_{i}(\mathbf{r}, t)\right\rangle}{\partial t}=\left[-\frac{1}{2} \nabla^{2}+U(\mathbf{r}, t)\right]\left|\psi_{i}(\mathbf{r}, t)\right\rangle .
$$

Before the voltage is applied, the $i$ th electron is characterized by its ground-state wavefunction, $\left|\psi_{i}^{0}(\mathbf{r})\right\rangle$, as follows:

$$
\left[-\frac{1}{2} \nabla^{2}+U_{\mathrm{g}}(\mathbf{r})\right]\left|\psi_{i}^{0}(\mathbf{r})\right\rangle=E_{i}\left|\psi_{i}^{0}(\mathbf{r})\right\rangle,
$$

with $E_{i}$ being its eigenenergy and $U_{g}$ the bulk potential energy. Consider a sinusoidal voltage with frequency $v$ applied to the electrode from $t=0$, i.e., $V(t)=V_{0} \cos (v t)$ with $V_{0}$ being the amplitude. It is widely accepted that the electrostatic potential is shifted homogeneously in real space, except for an extremely high $v$ [18-20]. Therefore, we have $U(r, t)=U_{\mathrm{g}}(r)+\Delta \cos (v t)$ with $\Delta=-e V_{0}$. $\left|\psi_{i}(\mathbf{r}, t)\right\rangle$ can be solved by an expansion approach similar to the time-dependent perturbation theory. Denote $\left|E_{i}\right\rangle \equiv$ $\exp \left(-\mathrm{i} E_{i} t\right)\left|\psi_{i}^{0}(\mathbf{r})\right\rangle$, which gives $\left|\psi_{i}(\mathbf{r}, t)\right\rangle$ for $\Delta=0$, and $\left|E_{i}+n v\right\rangle \equiv \exp \left[-\mathrm{i}\left(E_{i}+n v\right) t\right]\left|\psi_{i}^{0}(\mathbf{r})\right\rangle$, with $n$ being any integer number. Under the sinusoidal voltage, $\left|\psi_{i}(\mathbf{r}, t)\right\rangle$ can be formally expanded as follows:

$$
\left|\psi_{i}(\mathbf{r}, t)\right\rangle=\sum_{n=-\infty}^{\infty} c_{n}(t)\left|E_{i}+n v\right\rangle .
$$

Here the coefficients $\left\{c_{n}(t)\right\}$ are to be determined by inserting equation (4) into equation (2). Taking $\Delta \cos (v t)$ as the perturbative single-electron potential, we have

$$
\begin{aligned}
& \Delta \cos (v t)\left|E_{i}+n v\right\rangle=\frac{\Delta}{2}\left(\mathrm{e}^{\mathrm{i} v t}+\mathrm{e}^{-\mathrm{i} v t}\right)\left|E_{i}+n v\right\rangle \\
& =\frac{\Delta}{2}\left\{\mathrm{e}^{-\mathrm{i}\left[E_{i}+(n-1) v\right] t}\left|\psi_{i}^{0}(\mathbf{r})\right\rangle+\mathrm{e}^{-\mathrm{i}\left[E_{i}+(n+1) v\right] t}\left|\psi_{i}^{0}(\mathbf{r})\right\rangle\right\} \\
& =\frac{\Delta}{2}\left[\left|E_{i}+(n-1) v\right\rangle+\left|E_{i}+(n+1) v\right\rangle\right] .
\end{aligned}
$$

After some algebra, we arrive at the following coupled linear differential equations for the expansion coefficients $\left\{c_{n}(t)\right\}$ :

$$
\begin{aligned}
\mathrm{i} & \sum_{n=-\infty}^{\infty} S_{m, n} \dot{c}_{n}(t) \\
& =\sum_{n=-\infty}^{\infty}\left(\Delta S_{m, n+1} / 2+\Delta S_{m, n-1} / 2-n \nu S_{m, n}\right) c_{n}(t),
\end{aligned}
$$

where $S_{m, n} \equiv\left\langle E_{i}+m v \mid E_{i}+n v\right\rangle$, and $m$ runs over all integers. There is a simpler way to solve equation (2). Combining equations (2) and (3), we have

$$
\begin{aligned}
& \mathrm{i} \frac{\partial\left|\psi_{i}(\mathbf{r}, t)\right\rangle}{\partial t}=\left[-\frac{1}{2} \nabla^{2}+U(\mathbf{r}, t)\right]\left|\psi_{i}(\mathbf{r}, t)\right\rangle \\
& =\left[E_{i}+\Delta \cos (v t)\right] \psi_{i}(\mathbf{r}, t) \\
& \quad\left|\psi_{i}(\mathbf{r}, t)\right\rangle=\exp \left[-\mathrm{i} E_{i} t-\mathrm{i} \int_{0}^{t} \Delta \cos (v \tau) \mathrm{d} \tau\right]\left|\psi_{i}^{0}(\mathbf{r})\right\rangle .
\end{aligned}
$$

The difference between $\left|\psi_{i}(\mathbf{r}, t)\right\rangle$ and $\left|E_{i}\right\rangle$ is just a phase factor $\exp \left[-\mathrm{i} \Delta v^{-1} \sin (v t)\right]$, the same for all electrons (irrespective of $i$ ). In other words, all the eigenvalues $E_{i}$ are shifted by the same amount of energy $U(r, t)-U_{\mathrm{g}}(r)$ at time $t$ due to the applied voltage while the wavefunction remains the same except for an overall phase factor. Note that this conclusion holds for an arbitrary time-dependent voltage $V(t)$. Therefore, the electrode Fermi energy at any time $t$ can always be defined as $E_{\mathrm{f}}(t)=E_{\mathrm{f}}(0)-e V(t)$. Consequently, the bias voltage in our simulations is also time-dependent and is simply the difference between the applied potentials on the left and right electrodes.

Equation (1) is integrated in the time domain to evaluate the time-dependent current through the device depicted in figure 1. The fourth-order Runge-Kutta method is employed. In our calculations, the crystal structure of aluminum is used for the electrode, and the CNT segment is extracted from an ideal infinitely long tube without modification. Both ends of the $\mathrm{CNT}$ are connected to the $\mathrm{Al}(100)$ surface with a preset separation of $1.5 \AA$. We include explicitly in the simulation box $32 \mathrm{Al}$ atoms of the left electrode and $32 \mathrm{Al}$ atoms of the right electrode along with $60 \mathrm{C}$ atoms of the CNT where the ground-state Kohn-Sham Fock matrix of the extended system (including extra portions of leads of 16 atoms on each side) is calculated self-consistently by the conventional DFT method using the local density approximation (LDA) for the $\mathrm{XC}$ functional. A renormalization group method [21] is used to evaluate the surface Green's function of an isolated lead. In order to generate the correct surface Green's function and self-energies, it is important that the simulation box is large enough, so that the bulk properties are correctly reproduced 
near its two ends. This is achieved by including more $\mathrm{Al}$ atoms in the simulation box until the coupling matrix between two adjacent unit cells and the electrostatic potential does not vary near the two ends. For the time propagation calculations, we have verified that the transient current remains unchanged upon further inclusion of more $\mathrm{Al}$ atoms. The minimum basis set STO-3G is adopted. The time-dependent electric current $J$ through the left (right) electrode is obtained via the trace of the corresponding dissipative term $Q_{\mathrm{L}}\left(Q_{\mathrm{R}}\right)$ :

$$
J_{\alpha}(t)=-\operatorname{Tr}\left[Q_{\alpha}(t)\right] .
$$

The transient dynamics of the device $\mathrm{D}$ is solved by directly integrating the EOM (1) subject to boundary conditions at the left and right interfaces $\left(S_{\mathrm{L}}\right.$ and $\left.S_{\mathrm{R}}\right)$ of the simulation box, i.e., the induced Hartree potential $\delta v^{\mathrm{H}}(\mathbf{r}, t)$ inside the device region $\mathrm{D}$ satisfies the following Poisson equation and boundary condition:

$$
\begin{gathered}
\nabla^{2} \delta v^{\mathrm{H}}(\mathbf{r}, t)=-4 \pi \delta \rho_{\mathrm{D}}(\mathbf{r}, t) \\
\left.\delta v^{\mathrm{H}}(\mathbf{r}, t)\right|_{S_{\mathrm{L}}}=V_{\mathrm{L}}(t) \\
\left.\delta v^{\mathrm{H}}(\mathbf{r}, t)\right|_{S_{\mathrm{R}}}=V_{\mathrm{R}}(t) .
\end{gathered}
$$

The Poisson equation is solved using a multi-grid method [22]. Here $\delta \rho_{\mathrm{D}}(\mathbf{r}, t)=\rho_{\mathrm{D}}(\mathbf{r}, t)-\rho_{\mathrm{D}}(\mathbf{r}, 0)$ is the induced electron density for the device $\mathrm{D}$, and $V_{\mathrm{L}}(t)\left(V_{\mathrm{R}}(t)\right)$ is the external bias voltage applied to the left (right) electrode at time $t$. Since aluminum is a good conductor, the induced electrostatic potential is constant across the electrode, in particular for the region far away from the device. Therefore, in our calculations, the change in electrostatic potential is the same over the entire left or right electrode, and has the same amplitude as its time-dependent applied potential. This provides the boundary condition for solving the Poisson equation for the electronic dynamics of the device region. The same procedure has been widely adopted in treating time-dependent transport problems [18-20].

\section{Transient current and dynamic admittance}

Figures 2(a) and (b) show the current versus time for two different types of bias voltage switched on at $t=0$. In figure 2(a), the bias voltage $V_{\mathrm{b}}$ is turned on exponentially. We observe that the current reaches its steady state in $\sim 12 \mathrm{fs}$. The time-dependent current can be fitted by $I_{0}\left(1-\mathrm{e}^{-t / \tau}\right)$ with $\tau=2.8 \mathrm{fs}$ and $I_{0}=13.9 \mathrm{nA}$, leading to a characteristic time of $2.8 \mathrm{fs}$. The reason for such a fast switch-on time is that the transient dynamic process involves only electrons. We also considered the response to a sinusoidal bias voltage turned on at $t=0$. Figure 2(b) shows the corresponding time-dependent current. We see a phase delay in the current response to bias voltage. This implies at this frequency the device is overall inductive.

Figure 3(a) plots the potential energy change for an electron along the central axis at $t=0.02,1$, and $12 \mathrm{fs}$. The bias voltage is turned on exponentially, as shown in figure 2(a). The potential change is the sum of the applied potential and the potential caused by the induced charge. Our calculation leads to the following observations.

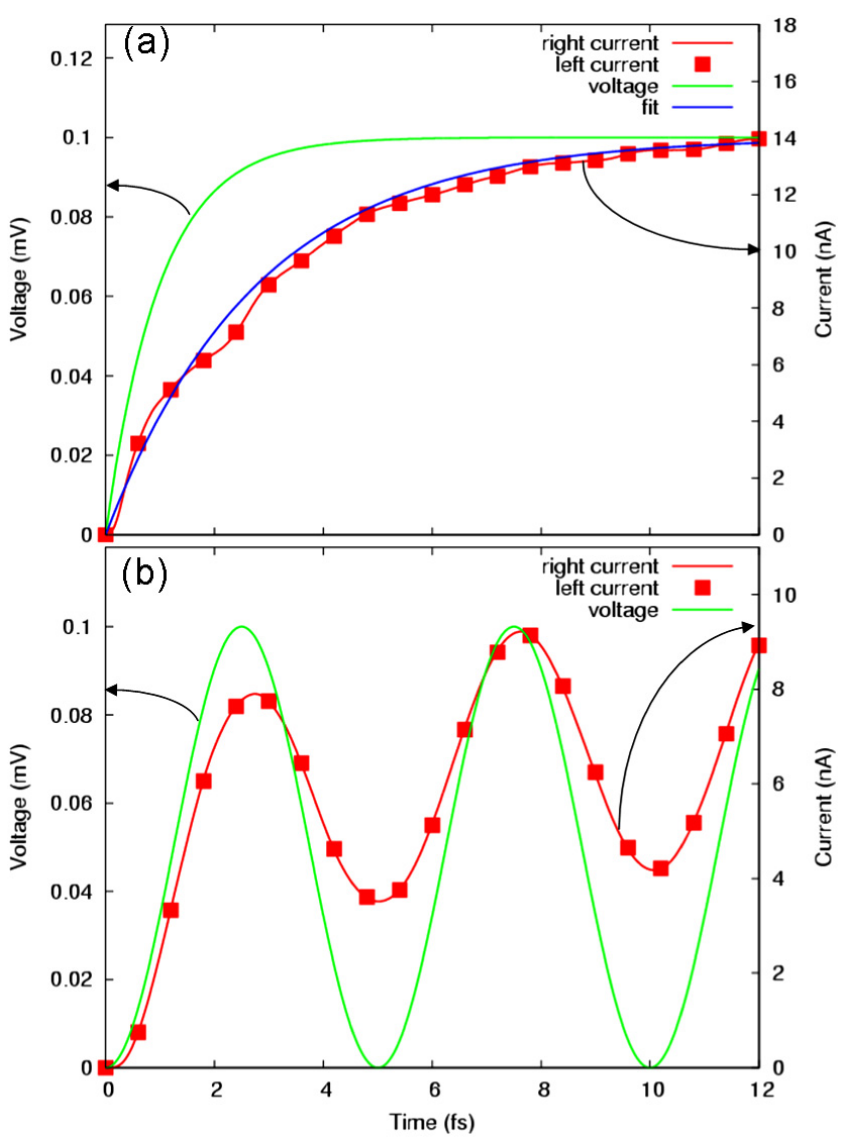

Figure 2. (a) and (b) Transient current (dark grey lines and squares) and applied bias voltage (green lines) for the Al-CNT-Al system. (a) The bias voltage is turned on exponentially, $V_{\mathrm{b}}=V_{0}\left(1-\mathrm{e}^{-t / a}\right)$ with $V_{0}=0.1 \mathrm{mV}$ and a time constant $a=1 \mathrm{fs}$. The darkest solid line in (a) is a fit to the transient current. (b) The bias voltage is sinusoidal with a period of $5 \mathrm{fs}$. The dark grey line is for current from the right electrode, and squares are current from the left electrode.

- After turning on the bias voltage, at $t=0.02 \mathrm{fs}$ the electrons have not yet responded to the applied voltage, and the external field is hardly screened, dropping uniformly across the entire Al-CNT-Al system.

- At $t=1 \mathrm{fs}$ the potential drop occurs mostly on the CNT since aluminum is more polarizable. It takes less than $1 \mathrm{fs}$ for the electrons on the $\mathrm{Al}$ electrodes to screen the applied potential.

- In figure 3(b) we plot the induced charge along Al$\mathrm{CNT}-\mathrm{Al}$ at $t=4 \mathrm{fs}$. Alternating positive and negative charge distributions on the CNT cancel each other so that its net induced charge is zero. The excess charge resides primarily at two interfaces and forms an effective capacitor, as depicted schematically in figure 3(c).

The Al-CNT-Al system depicted in figure 1 has central inversion symmetry. As a consequence, there is no net charging for the device, and the overall time-dependent current is conserved [23]. This was confirmed by our numerical simulation. The current entering the system has the same magnitude as the current leaving, as shown in figures 2(a) and (b). Therefore, the admittance matrix element $G_{\alpha \beta}(\omega)$ 


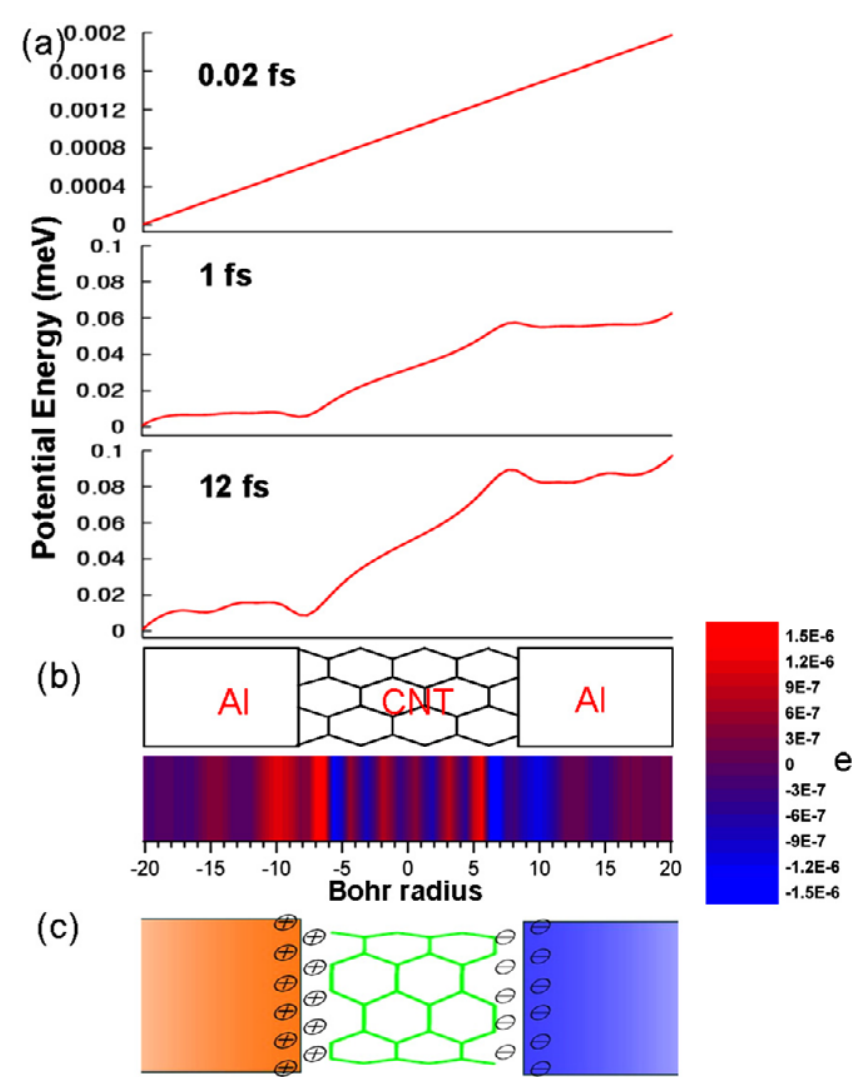

Figure 3. (a) Induced electrostatic potential energy distribution along the central axis at $t=0.02,1$ and $12 \mathrm{fs}$. (b) Induced charge distribution along Al-CNT-Al at $t=4 \mathrm{fs}$. The bias voltage is turned on exponentially. (c) Schematic diagram showing the induced charge accumulation at two interfaces, forming an effective capacitor.

$\left(\alpha, \beta=\mathrm{L}\right.$ or R) satisfies $G_{\mathrm{LL}}=G_{\mathrm{RR}}=-G_{\mathrm{LR}}=-G_{\mathrm{RL}}=$ $G(\omega)[23,24]$. Taking the Fourier transform, the simulated transient dynamics leads to $I(\omega)$ and $V(\omega)$, from which we obtain the dynamic admittance $G(\omega)=I(\omega) / V(\omega)$. We find that both types of bias voltage lead to essentially the same dynamic admittance. This implies that the electrical response is in the linear response regime and also validates the accuracy of our calculations. Figure 4 shows the real and imaginary parts of the resulting dynamic admittance.

\section{Equivalent circuit}

Now the question is how to model the Al-CNT-Al device. Our above results and analysis show that our molecular device has both inductive and capacitive components. As the current enters into the device region from the left electrode, a part of it, $I_{C}$, charges the left interface (see figure 5(a)). The remaining current, $I_{\mathrm{L}}$, goes straight through the device and is joined by $I_{C}$ at the right interface. Therefore, our device can be modeled by the classical circuit depicted in figure 5(b). At zero frequency, the steady current goes only through the $R-L$ branch. $R_{\mathrm{L}}$ is simply the steady state resistance $[25,26]$, which is calculated to be $7.39 \mathrm{k} \Omega$. At a high frequency, the $R-L$ branch is blocked due to the inductor, and the ac current primarily goes through the $R-C$ branch. A similar circuit was proposed to fit the

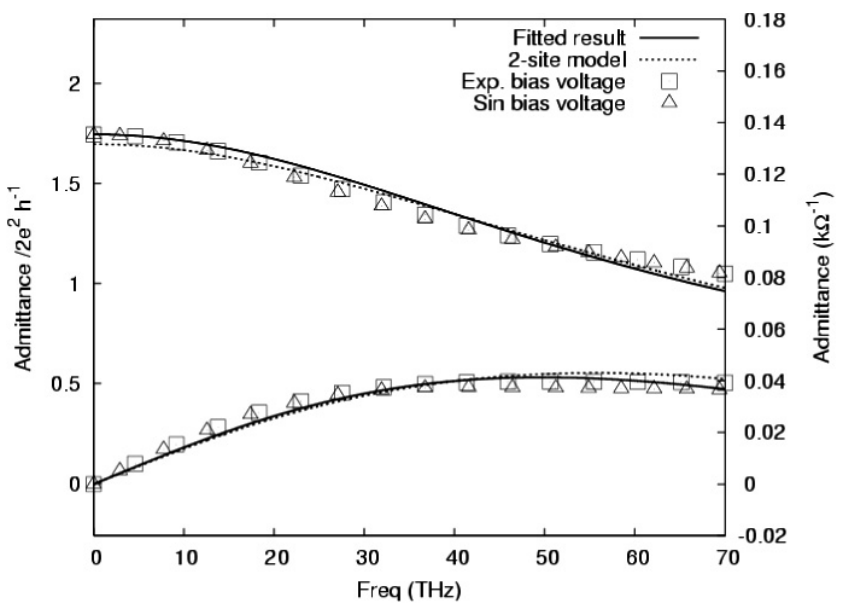

Figure 4. Dynamic admittance calculated with an exponential bias voltage turned on at $t=0$ (open squares) and a sinusoidal bias voltage (open triangle) turned on at $t=0$. The solid lines show the results fitted to the classical circuit. The dotted lines are the admittance of the two-site model system. The upper curves are the real part of the admittance while the lower ones are the imaginary part.

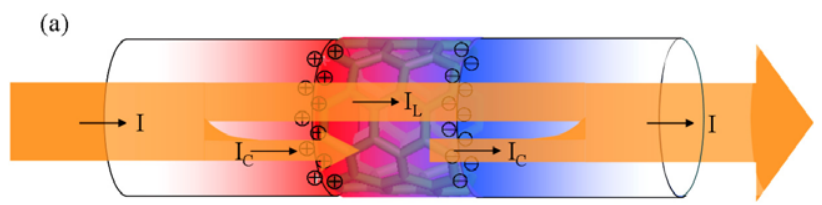

(b)

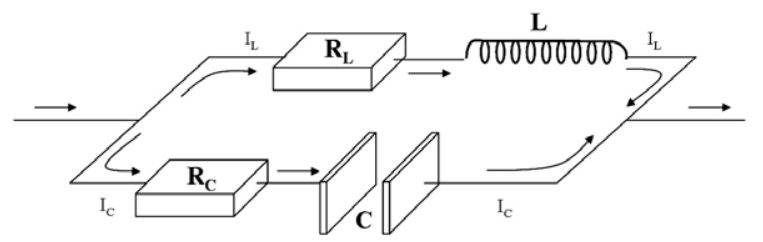

Figure 5. (a) Current flow in the parallel circuit. (b) The equivalent electric circuit. $R_{\mathrm{L}}=7.39 \mathrm{k} \Omega, L=16.6 \mathrm{pH}, R_{C}=6.45 \mathrm{k} \Omega$, and $C=0.073 \mathrm{aF}$

numerical dynamic admittance of a model quantum wire [27] with an additional inductor to account for a resonance at a high frequency.

Büttiker and co-workers [10] studied a mesoscopic capacitor made of two plates with each coupled to an electron reservoir via a narrow lead. They discovered that the charge relaxation resistance $R_{C}$ is universal, independent of the transmission details, leading to $R_{C}=R_{1}+R_{2}$ with $R_{1}$ and $R_{2}$ given by

$$
R_{\alpha}=\frac{h}{2 e^{2}} \frac{\sum_{n} D_{\alpha, n}\left(E_{\mathrm{F}}\right)^{2}}{\left(\sum_{n} D_{\alpha, n}\left(E_{\mathrm{F}}\right)\right)^{2}}
$$

where $D_{\alpha, n}\left(E_{\mathrm{F}}\right)$ is the density of states (DOS) at the Fermi energy for the $n$th spin-specific charging channel of plate $\alpha$ $(\alpha=1,2)$, and $\sum_{n}$ is over all charging channels for $\alpha$. This has been confirmed by a recent experiment [28].

In our Al-CNT-Al system, the two interfaces correspond to the two plates of the capacitor (see figure 3(c)) and couple 


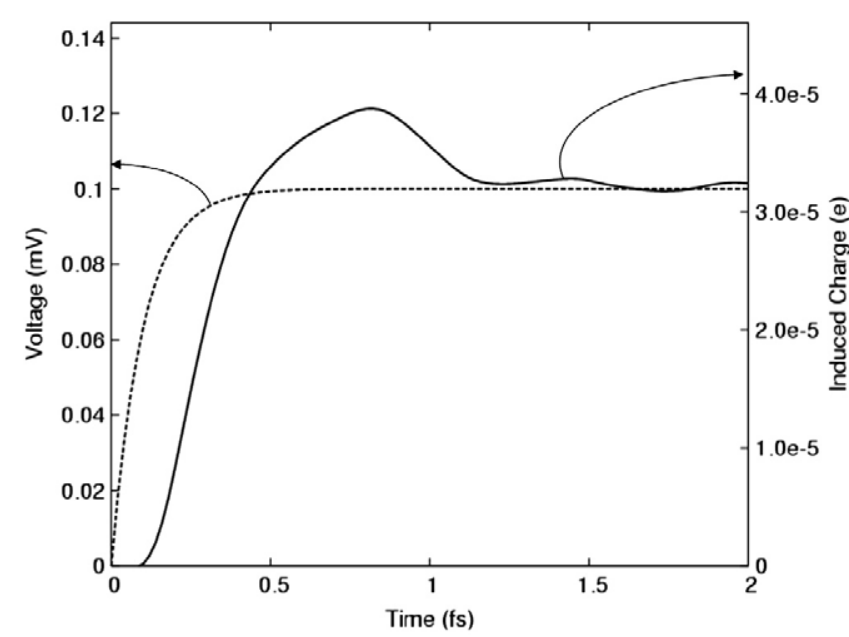

Figure 6. Applied voltage $V_{\mathrm{b}}$ (dotted line) and induced charge (solid line) versus time for a swiftly turned on bias voltage. $V_{\mathrm{b}}=V_{0}\left(1-\mathrm{e}^{-t / a}\right)$ with $V_{0}=0.1 \mathrm{mV}$ and $a=0.1 \mathrm{fs}$.

to the electrodes via $\mathrm{Al}$ leads. Our CNT has two degenerate orbitals for transmission, and both spin-up and spin-down electrons contribute to the transmission associated with each orbital. Therefore, there are four charging channels for each interface. According to equation (10), the charge relaxation resistance for the Al-CNT-Al system is

$$
R_{C}=\frac{h}{2 e^{2}} \times \frac{1}{4}+\frac{h}{2 e^{2}} \times \frac{1}{4}=\frac{h}{4 e^{2}} .
$$

We tune the values of $L$ and $C$ to fit the calculated dynamic admittance while fixing $R_{C}=\frac{h}{4 e^{2}}$ and $R_{L}=7.39 \mathrm{k} \Omega$. The resulting values of $L$ and $C$ are $16.6 \mathrm{pH}$ and $0.073 \mathrm{aF}$, respectively. In figure 4 the solid lines depict the real and imaginary parts of $G(\omega)$ fitted by the equivalent electric circuit, which agree well with our TDDFT simulation up to $70 \mathrm{THz}$. As the bias voltage is turned on, the induced charge starts to accumulate at the two interfaces, with a characteristic charging time $\tau_{C}=R_{C} C=0.47 \mathrm{fs}$ for the $R-C$ branch. To confirm this, we turn on the bias voltage swiftly, as indicated in figure 6 , where the induced charge versus time is plotted. It is estimated from the solid line that the charging time is $\sim 0.5 \mathrm{fs}$, which is consistent with the value of $\tau_{C}$. We can also estimate the capacitance $C$ directly from the excess charge $Q$ at the interfaces. For bias voltage $V_{\mathrm{b}}=0.1 \mathrm{mV}$, we find that at the steady state $Q$ is roughly $3 \times 10^{-5} e$. Therefore, $C$ is estimated to be

$$
C=Q / V_{\mathrm{b}}=0.05 \mathrm{aF},
$$

which is of the same order of magnitude as the calculated $C=$ $0.073 \mathrm{aF}$. The quantitative discrepancy is due to the uncertainty in defining the excess charge at the interfaces. According to equations (15) and (17) below, the inductance $L$ is $\sim\left(\frac{h}{\gamma}\right) R_{L}$, where $\gamma$ is the coupling strength between the device and an electrode. The average line width $\langle\gamma / 4\rangle$ at the Fermi energy is $\sim 0.38 \mathrm{eV}$. Thus, $\left(\frac{h}{\gamma}\right) R_{L} \approx 18.8 \mathrm{pH}$. This is close to the calculated $L=16.6 \mathrm{pH}$.

We have carried out TDDFT simulations on other CNTs with different lengths and found that their electrical responses

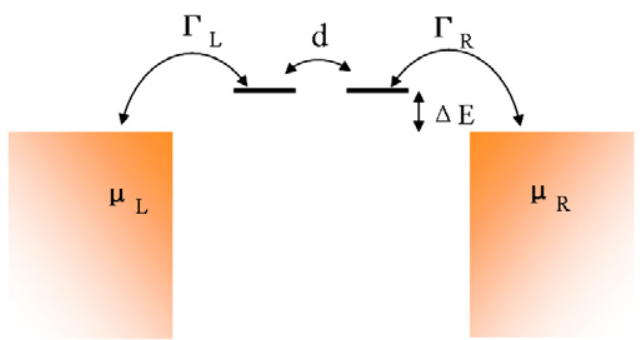

Figure 7. A two-site system coupled to the left and right electrodes. $\Gamma_{L}=\Gamma_{\mathrm{R}}=\gamma / 2$.

Table 1. Values of $L, C, R_{L}$ and $R_{C}$ in the equivalent circuit for different CNTs.

\begin{tabular}{lccl}
\hline & $60 \mathrm{C}$ atoms & $40 \mathrm{C}$ atoms & $20 \mathrm{C}$ atoms \\
\hline$L(\mathrm{pH})$ & 16.6 & 9.77 & 4.76 \\
$C(\mathrm{aF})$ & 0.073 & 0.06 & 0.06 \\
$R_{L}(\mathrm{k} \Omega)$ & 7.39 & 10.76 & 4.81 \\
$R_{C}(\mathrm{k} \Omega)$ & 6.45 & 6.45 & 6.45 \\
\hline
\end{tabular}

can be reproduced by the same type of circuit as in figure 5(b). Their $R_{L}, R_{C}, L$ and $C$ values are given in table 1 . These circuit components do not show a clear trend with the length of the CNT. This is due to the quantum size effect. The proposed equivalent circuit is limited to the linear response of two-terminal molecular electronic devices. Beyond the linear regime, nonlinear electric components are required to reproduce the nonlinear electrical responses of molecular devices.

\section{Equivalent circuit of two-site model system}

To further confirm the equivalent electric circuit for our CNTbased molecular conductor, we designed a simple model: a two-site system in contact with the left and right electrodes (figure 7). The two sites are degenerate in energy $\varepsilon_{0}$ and the dynamic admittance can be calculated using equation (12) below, where $d$ and $\gamma$ are the couplings between the two sites and between electrodes and the site, respectively. $\Delta E$ is the energy difference of the sites and the electrodes. The two-site device is employed to model general two-terminal molecular devices. The two sites are used to approximate the many atomic orbitals in general molecular devices. The coupling between the two sites represents the interactions among these atomic orbitals, and the coupling between the left (right) electrode and the first (second) site represents the interaction between the left (right) electrode and the left (right) end of the molecular device. One of the level couples to the left electrode with $\Gamma_{\mathrm{L}}$, and the other couples to the right electrode with $\Gamma_{\mathrm{R}}$. We set $\Gamma_{\mathrm{L}}=\Gamma_{\mathrm{R}}=\gamma / 2$. We find that the dynamic admittance of our CNT device in figure 4 can indeed be reproduced by the two-site model. The dotted lines are the real and imaginary parts of the dynamic admittance of the two-site model by setting $d=0.193 \mathrm{eV}, \gamma=0.785 \mathrm{eV}$, and $\Delta E=0.175 \mathrm{eV}$. This demonstrates that the simple two-site device can be used 
to model the electric response of our CNT devices:

$$
\begin{aligned}
G(\omega) & =\frac{4 e^{2}}{h} \int \mathrm{d} \varepsilon F(\varepsilon, \omega) \operatorname{Tr}\left\{G_{\mathrm{eq}}^{r}(\varepsilon+\hbar \omega) \hat{\Gamma}_{L} G_{\mathrm{eq}}^{a}(\varepsilon) \hat{\Gamma}_{\mathrm{R}}\right\} \\
= & \frac{4 e^{2}}{h} \int \mathrm{d} \varepsilon F(\varepsilon, \omega)\left\{4 d^{2} \gamma^{2}-(\hbar \omega)^{2} \gamma^{2}\right. \\
& \left.-4 \mathrm{i} \gamma \hbar \omega\left[(\varepsilon-\Delta E)(\varepsilon-\Delta E+\hbar \omega)+d^{2}+\gamma^{2} / 16\right]\right\} \\
& \times\left\{16\left[(\varepsilon-\Delta E-\mathrm{i} \gamma / 4)^{2}-d^{2}\right]\right. \\
& \left.\times\left[(\varepsilon-\Delta E+\hbar \omega-\mathrm{i} \gamma / 4)^{2}-d^{2}\right]\right\}^{-1}
\end{aligned}
$$

where $F(\varepsilon, \omega)=\frac{f(\varepsilon)-f(\varepsilon+\hbar \omega)}{\hbar \omega}$.

To simplify the discussion, we set $\varepsilon_{0}=\mu_{\mathrm{L}}=\mu_{\mathrm{R}}$ or $\Delta E=0$. Near the capacitor limit where $d=\frac{\gamma}{4} \eta, 0<\eta \ll 1$, we derive the frequency-dependent conductance analytically, as follows:

$$
\begin{aligned}
G(\omega) & =\frac{2 e^{2}}{h} 4 \eta^{2}-\mathrm{i} \omega \frac{2 e^{2}}{h} \frac{4 \hbar}{\gamma}\left(1-9 \eta^{2}\right) \\
+ & \omega^{2} \frac{2 e^{2}}{h}\left(\frac{4 \hbar}{\gamma}\right)^{2}+\mathrm{O}\left(\omega^{2} \eta^{2}\right) .
\end{aligned}
$$

Expanding the dynamic conductance of the electric circuit in figure $5(\mathrm{~b})$ in $\omega$ leads to a dynamic conductance:

$G(\omega)=\frac{1}{R_{L}}+\mathrm{i} \omega\left(-C+\frac{L}{R_{L}^{2}}\right)+\omega^{2}\left(R_{C} C^{2}-\frac{L^{2}}{R_{L}^{3}}\right)+\mathrm{O}\left(\omega^{3}\right)$.

Comparing equations (13) and (14), we find that the dynamic electric response of the two-site device can indeed be mapped to the same equivalent circuit in figure 5(b), and

$$
\begin{array}{cl}
R_{L}=\frac{h}{2 e^{2}} \frac{1}{4 \eta^{2}}, & L=\left(\frac{9 \hbar}{\gamma}\right) R_{L}, \\
R_{C}=\frac{h}{2 e^{2}}, & C=\frac{2 e^{2}}{h} \frac{4 \hbar}{\gamma} .
\end{array}
$$

Near the ballistic transport limit where $d=\frac{\gamma}{4}(1 \pm \delta)$ and $0<\delta \ll 1$, which is similar to our system, the dynamic conductance is

$$
\begin{aligned}
G(\omega) & =\frac{2 e^{2}}{h}+\mathrm{i} \omega \frac{2 e^{2}}{h} \frac{2 \hbar}{\gamma}(1+\delta) \\
- & \omega^{2} \frac{2 e^{2}}{h}\left(\frac{2 \hbar}{\gamma}\right)^{2}\left(1+\frac{10}{3} \delta\right)+\mathrm{O}\left(\omega^{3}\right) .
\end{aligned}
$$

Comparing equation (16) with equation (14), we find that

$$
\begin{gathered}
R_{L}=\frac{h}{2 e^{2}}, \\
L=\left(\frac{2 \hbar}{\gamma}\right) R_{L}\left(1+\frac{5}{3} \delta\right)=\frac{2 \hbar^{2} \pi}{e^{2} \gamma}\left(1+\frac{5}{3} \delta\right), \\
R_{C}=\frac{h}{2 e^{2}}, \quad C=\frac{4}{3} \frac{\hbar}{\gamma} R_{C}^{-1} \delta=\frac{4}{3 \pi} \frac{e^{2}}{\gamma} \delta .
\end{gathered}
$$

The above calculation shows that near both the capacitive and the inductive limits the electrical response of a two-site system can be modeled by the classical circuit in figure 5(b), and its charging relaxation resistance $R_{C}$ is $\frac{h}{4 e^{2}}+\frac{h}{4 e^{2}}=\frac{h}{2 e^{2}}$, which agrees with equation (10). If each site is two-fold degenerate, the charge relaxation resistance is reduced to half this value, i.e. $R_{C}=h / 4 e^{2}$, which is exactly the same as for our Al-CNT-Al system. Therefore, we have confirmed the equivalent circuit found for the CNT molecular device.

Büttiker and co-workers [10] have shown that charge relaxation resistance $R_{C}$ depends only on the number of transmission channels or transverse modes [2], $M$. This is supported by the two-site model and finite HOMO-LUMO gap. With each of the two sites having a degeneracy of two, the two-site model has two transmission channels and the analytical calculation shows that its charge relaxation resistance is exactly the same as that of our simulated nanotubes, $h / 4 e^{2}$ or $6.45 \mathrm{k} \Omega$. It is important to point out that the resistance for the steady state current, $R_{L}$, depends not only on the number of transmission channels or transverse modes but also on the reflectance at the interfaces between the device and the electrodes. It is the reflectance at the interfaces (not the finite size of the device) that leads to the deviation of $R_{L}$ from the exact quantized values, $h /\left(2 e^{2} M\right)$. The charge relaxation resistance, $R_{C}$, does not depend on the reflection or transmission at the interfaces at all [10].

\section{Discussion and conclusion}

Time-dependent quantum transport of CNT has been studied using the finite difference time domain method [29]. However, an effective mass model was introduced for the conduction band structure in CNTs, and Schottky barriers were introduced to model the interaction between the electrodes and the CNT, and thus, the atomistic details were lacking in [29]. Our quantum mechanical simulation is one of first principles, and it includes all electrons and full atomistic details. In our calculation, we solve the Poisson equation instead of the Maxwell equation. This is justifiable since we calculate the dynamic admittance up to $70 \mathrm{THz}$. At this frequency, the corresponding electromagnetic wavelength is $4283 \mathrm{~nm}$, which is much longer than the size of our device $(\sim 2 \mathrm{~nm})$.

An $R-L$ circuit was proposed for a long CNT with $L$ as the kinetic inductance $[5,8,9]$. In the presence of a substrate, extra parallel capacitors are introduced between the tube and substrate $[8,9]$. When a CNT sits on top of an electrode via van der Waals attraction, an effective capacitor is introduced between the CNT and electrode in addition to a parallel contact resistor [5]. The kinetic inductance and quantum capacitance of a long CNT are intrinsic properties of the tube, being determined by the DOS at the Fermi energy or the Fermi velocity $v_{\mathrm{f}}$. In our case, the CNT is much shorter, and is welded to the electrodes covalently. The electrical responses of the interfaces and tube cannot be separated. We find that the capacitance $C$ of this system is determined mostly by the local charges or DOS at interfaces. As the length of the CNT increases, the capacitance due to the interfaces decreases and the kinetic inductance of the tube dominates. As a result, our equivalent circuit reduces to the $R-L$ branch only, which is consistent with the equivalent electric circuit proposed for long CNTs $[5,8,9]$. The systems studied here are symmetric and there are no charge accumulations, which ensures that the left and right currents are always the same in magnitude. When 
there is charge accumulation, the currents going in and coming out can be different. In such a case, an extra capacitor may be introduced in the equivalent circuit. Wang and co-workers [30] introduced the dwell time $\tau_{\mathrm{d}}$ to unify the inductance expression for short and long tubes as $L \sim \tau_{\mathrm{d}} \frac{h}{e^{2}}$. For a long 1D system of length $l, \tau_{\mathrm{d}} \sim l / v_{\mathrm{f}}$, leading to an expression for the kinetic inductance per length $L / l \sim \frac{h}{v_{\mathrm{f}} e^{2}}$.

The two-site model is designed to represent generic coherent multi-atom electronic devices. Therefore, we may generalize our findings on the equivalent electric circuit to other two-terminal molecular devices, and conclude that the linear electric response of any coherent two-terminal molecular electronic devices can be modeled by the classical circuit depicted in figure 5(b) as long as the net charges on these systems are conserved at any time. When the charge in the device fluctuates in time, an extra capacitor needs to be introduced to satisfy Kirchhoff's current law. Our proposed equivalent circuit is limited to the linear responses of two-terminal molecular electronic devices. Beyond the linear regime, nonlinear electric components are required to reproduce the nonlinear electric responses of molecular devices.

To summarize, our nanoscale device has very small values of $L$ and $C$, leading to the short switching time. Such a fast switching speed for electronic devices based on nanometersized CNTs indicates that these devices will not limit switching speeds in the foreseeable future. The equivalent electric circuit of the parallel $R-L$ and $R-C$ circuit in figure 5(b) is not limited to the CNT-based conductor studied in this work. It also applies to other coherent two-terminal symmetric molecular, nanoscopic and mesoscopic electronic devices. $R_{L}$ is given by the Landauer-Büttiker formula for steady state current $[25,26]$, and $R_{C}$ is the universal charge relaxation resistance depending only on the number of charging channels and spin polarization [10]. $L$ is determined by the dwell time of the electrons inside the device as $L \sim \tau_{\mathrm{d}} \frac{h}{e^{2}}$ [30]. $C$ is the electrochemical capacitance, which is determined by the geometry and the DOS at interfaces $[10,30]$. These results should be useful in designing nanoscale electronics systems required over the next decade.

\section{Acknowledgments}

The authors thank Hong Guo, Jian Wang, and YiJing Yan for stimulating discussions. The Caltech team was supported partly by Intel Components Research (Portland, OR) and by NSF (CCF-0524490). The HKU team was supported by the Hong Kong Research Grant Council (HKU 7011/06P, N_HKU 764/05, HKUST 2/04C).

\section{References}

[1] Aviram A and Ratner M A 1974 Chem. Phys. Lett. 29277

[2] Datta S 1995 Electron Transport in Mesoscopic Systems (Cambridge: Cambridge University Press)
[3] Taylor J, Guo H and Wang J 2001 Phys. Rev. B 63245407

[4] Xiang J, Lu W, Hu Y, Wu Y, Yan H and Lieber C M 2006 Nature $\mathbf{4 4 1} 489$

[5] Plombon J J, O’Brien K P, Gstrein F, Dubin V M and Jiao Y 2007 Appl. Phys. Lett. 90063106

Gomet-Rojas L, Bhattacharyya S, Mendoza E, Cox D C, Rosolen J M and Silva S R P 2007 Nano Lett. 72672

[6] Kim Y H, Tahir-Kheli J, Schultz P A and Goddard W A III 2006 Phys. Rev. B 73235419

[7] Javey A, Guo J, Paulsson M, Wang Q, Mann D, Lundstrom M and Dai H J 2004 Phys. Rev. Lett. 92106804

[8] Burke P J 2003 IEEE Trans. Nanotechnol. 255

Li S, Yu Z, Yen S-F, Tang W C and Burke P J 2004 Nano Lett. 4753

[9] Raham A, Guo J, Datta S and Lundstrom M S 2003 IEEE Trans. Electron Devices $\mathbf{5 0} 1853$

[10] Büttiker M, Thomas H and Pretre A 1993 Phys. Lett. A 180364

Blanter Y M, Hekking F W J and Büttiker M 1998 Phys. Rev. Lett. 811925

[11] Burke K, Car R and Gebauer R 2005 Phys. Rev. Lett. 94146803

[12] Ke S-H, Baranger H U and Yang W 2004 Phys. Rev. B 70085410

[13] Yoon Y-G, Delaney P and Louie S G 2002 Phys. Rev. B 66073407

[14] Di Carlo A, Gheorghe M, Lugli P, Sternberg M, Seifert G and Frauenheim T 2002 Physica B 31486

[15] Zheng X, Chen G H, Li Z B, Deng S Z and Xu N S 2004 Phys. Rev. Lett. 92106803

[16] Zheng X, Wang F, Yam C Y, Mo Y and Chen G H 2007 Phys. Rev. B 75195127

[17] Maciejko J, Wang J and Guo H 2006 Phys. Rev. B 74085324

[18] Jauho A-P, Wingreen N S and Meir Y 1994 Phys. Rev. B 505528

[19] Li X Q and Yan Y J 2007 Phys. Rev. B 75075114

[20] Kurth S, Stefanucci G, Almbladh C O, Rubio A and Gross E K U 2005 Phys. Rev. B 72035308

[21] López Sancho M P, López Sancho J M and Rubio J 1985 J. Phys. F: Met. Phys. 15851

[22] Adams J 1989 Appl. Math. Comput. 34113

[23] Büttiker M, Pretre A and Thomas H 1993 Phys. Rev. Lett. 704114

Büttiker M, Pretre A and Thomas H 1993 Phys. Rev. Lett. 71465

Wang B, Wang J and Guo H 1999 Phys. Rev. Lett. 82398

[24] Fu Y and Dudley S C 1993 Phys. Rev. Lett. 7065

Fu Y and Dudley S C 1993 Phys. Rev. Lett. 71466

[25] Landauer R 1957 IBM J. Res. Dev. 1223

Landauer R 1970 Phil. Mag. 21863

[26] Büttiker M and Imry Y 1985 J. Phys. C: Solid State Phys. 18 L467

Büttiker M, Imry Y, Landauer R and Pinhas S 1985 Phys. Rev. B 316207

[27] Cunibert G, Sassetti M and Kramer B 1998 Phys. Rev. B 571515

[28] Gabelli J, Feve G, Berroir J-M, Placais B, Cavanna A, Etienne B, Jin Y and Glattli D C 2006 Science 313499

[29] Chen Y, Ouyang Y, Guo J and Wu T X 2006 Appl. Phys. Lett. 89203122

[30] Wang J, Wang B G and Guo H 2007 Phys. Rev. B 75155336 